\title{
Atributos dendrométricos, estructura poblacional y diversidad de estadios de Dioon holmgrenii (Cycadales: Zamiaceae)
}

\author{
Mario Valerio Velasco-García, Juan Ignacio Valdez-Hernández, Carlos Ramírez-Herrera* \\ \& María Luisa Hernández-Hernández \\ Postgrado en Ciencias Forestales Colegio de Postgraduados, Campus Montecillo. Montecillo, Estado de México, \\ México; taxodium01@hotmail.com, ignaciov@colpos.mx, kmcramcolpos@gmail.com, merry57_hdz@hotmail.com \\ * Correspondencia
}

Recibido 30-IV-2017. Corregido 24-VII-2017. Aceptado 24-VIII-2017.

\begin{abstract}
Forest characteristics, population structure and life-stages diversity of Dioon holmgrenii (Cycadales: Zamiaceae). Dioon holmgrenii is an endangered species and found in Southern Oaxaca, Mexico. Notwithstanding the extintion condition of this species, there is no relevant information on the ecological aspects of its populations, and this situation complicates the approval of management plans for its conservation. Our aim was to study $D$. holmgrenii forest characteristics, population structure and life-stages diversity in ten populations located in Sierra Madre del Sur, Oaxaca. For this, four sampling units (SU) measuring 25 x $50 \mathrm{~m}$ each $\left(1250 \mathrm{~m}^{2}\right)$ were established per population, $40 \mathrm{SU}$ in total $\left(50000 \mathrm{~m}^{2}\right)$. Basal diameter (BD), basal area (BA), stem height (AT), crown diameter (DC), crown area (AC), and number of leaves (NH), were measured for each individual, from January to May 2013. Additionally, plant density, population structure, structural dominance index (IDE), heterogeneity (H'), evenness (E) and similarity (ISct) of life-stages (seedling, juvenile 1, juvenile 2, pre-reproductive, adult 1 , adult 2, adult 3 , and adult 4) were assessed. Plant density ranged from 148 to 954 individuals $\mathrm{ha}^{-1}$. Total basal area ranged from 1.3 to $17.6 \mathrm{~m}^{2}$.ha-1, and total crown area varied from 78.3 to 1 $136.3 \mathrm{~m}^{2} \cdot \mathrm{ha}^{-1}$. Forest traits of each individual $\left(\mathrm{DB}=7.7\right.$ to $13.4 \mathrm{~cm}, \mathrm{AB}=0.010$ to $0.023 \mathrm{~m}^{2}, \mathrm{AT}=0.20$ to $0.47 \mathrm{~m}$, $\mathrm{DC}=0.49$ to $1.09 \mathrm{~m}, \mathrm{AC}=0.55$ to $1.55 \mathrm{~m}^{2}$ and $\mathrm{NH}=7.06$ to 14.90$)$ were different $(\mathrm{p}<0.05)$ among populations. The highest values of IDE ( 0.01 to 5.63) were found in adult 1 and adult 2 in all populations. The most common population structure was found in those populations with the lowest proportions in juvenile 1 and juvenile 2 , and the highest proportion in adult 1 . Heterogeneity $\left(H^{\prime}=1.05\right.$ to 1.71$)$ and uniformity $(E=0.57$ to 0.88$)$ of life-stages were significantly different among populations $(\mathrm{p}<0.05)$, and at least four life-stages $(3.91=48.9 \times 8 / 100)$ were different among populations for half of the comparisons $(22$ of 45$)$ of similarity (ISct $<0.68)$. Alfa diversity of life-stages was high in populations without plant damage, while beta diversity was low in those populations with human presence. Life-stages with the highest reproductive vigour (adult 1, adult 2) showed the highest structural dominance in all populations. These results may support the design and operation of management plans of D. holmgrenii populations, to enhance its protection in the area. Rev. Biol. Trop. 65 (3): 1609-1624. Epub 2017 September 01.
\end{abstract}

Key words: basal and crown area, density, structural dominance, heterogeneity, uniformity, similarity of life-stages.

El orden Cycadales es un grupo ancestral de plantas con semillas que se originaron en el periodo Pérmico y posiblemente en el Carbonífero de la era Paleozoica, hace 280 a 345 Ma (Mamay, 1976; Zhifeng \& Thomas, 1989; Condamine, Nagalingum, Marshall, \& Morlon, 2015). Este orden, actualmente, consta de dos familias (Cycadaceae y Zamiaceae), 10 géneros y 331 especies (Christenhusz et al., 2011; Osborne, Calonje, Hill, Stanberg, L., \& Stevenson, 2012). La familia Zamiaceae se originó al final del Pérmico de la era Paleozoica, hace $258 \mathrm{Ma}$, mientras que el género Dioon Lindl. apareció en el Cretácico inferior de la era Mesozoica hace 144 Ma (Dawson, 1983; Condamine et al., 2015). 
México ocupa el segundo lugar en diversidad de cícadas con 54 especies de los géneros Ceratozamia Brongn., Dioon y Zamia L., correspondientes a la familia Zamiaceae, de las cuales $88.9 \%$ son endémicas (Osborne et al., 2012; Nicolalde-Morejón et al., 2014). La destrucción del hábitat ocasionada por cambio de uso de suelo, comercio ilegal e incendios son las causas por que la mayoría de las especies mexicanas estén amenazadas o en peligro de extinción (Vovides \& Iglesias, 1994; SEMARNAT, 2010; Lázaro-Zermeño, González-Espinoza, Mendoza, \& MartínezRamos, 2012; Nicolalde-Morejón et al., 2014; Velasco-García et al., 2016).

Dioon holmgrenii De Luca, Sabato \& Vázq. Torres es endémica de la Sierra Madre del Sur y Planicies Costeras del Pacífico (sensu Ortíz-Pérez, Hernández-Santana, \& Figueroa, 2004) en el estado de Oaxaca, México (VelascoGarcía et al., 2016). La mayoría de las poblaciones de $D$. holmgrenii están fragmentadas y aisladas debido a incendios forestales y cambio de uso de suelo para la agricultura temporal y ganadería intensiva, lo que ha provocado cambios en la estructura poblacional, heterogeneidad de estadios y en la dispersión espacial (Velasco-García et al., 2011; Velasco-García et al., 2016). Por lo anterior, D. holmgrenii está en peligro de extinción (SEMARNAT, 2010; Chemnick, Gregory, \& Morales, 2010) y su comercialización está restringida por CITES.

El conocimiento de la situación de las poblaciones de $D$. holmgrenii es necesario para diseñar planes de manejo para su conservación. En este sentido, el efecto de las perturbaciones y el cambio de uso de suelo sobre D. holmgrenii se estudiaron por Velasco-García et al. (2016). Sin embargo, aspectos dasométricos de las poblaciones, diversidad de estadios en cada población y la semejanza entre las mismas no se conocen. Esta información es útil para tomar decisiones y para diseñar estrategias de conservación para la especie. Con base en lo anterior, el objetivo de esta investigación fue determinar los atributos dendrométricos, estructurales y la diversidad de estadios de 10 poblaciones de D. holmgrenii. Las hipótesis planteadas fueron:
(1) los valores de los atributos dendrométricos son iguales entre las poblaciones, (2) las curvas de densidad relativa y la diversidad de los estadios son iguales entre las poblaciones.

\section{MATERIALES Y MÉTODOS}

Área de estudio y muestreo: El estudio se realizó en 10 poblaciones de Dioon holmgrenii ubicadas $\left(16^{\circ} 35^{\prime} 42^{\prime \prime}\right.$ - 15 $5^{\circ} 53^{\prime} 52.1^{\prime \prime} \mathrm{N} \&$ $97^{\circ} 51$ '35" - 9645'46.4” W) en la Sierra Madre del Sur y Planicies Costeras del Pacífico en el estado de Oaxaca, México (Velasco-García et al., 2016). Cuatro unidades de muestreo (UM) contiguas (Pérez-Farrera, Quintana-Ascencio, \& Salvatierra-Izaba, 2000; Pérez-Farrera et al., 2006; Octavio-Aguilar, González-Astorga, \& Vovides, 2008) de $25 \times 50 \mathrm{~m}\left(1250 \mathrm{~m}^{2}\right) \mathrm{se}$ establecieron en cada población, donde evaluaciones dendrométricas, estructurales y la diversidad de estadios se realizaron de enero a mayo del año 2013.

Atributos dendrométricos de las poblaciones: Todas las plantas de Dioon holmgrenii se enumeraron en las UM para conocer la densidad (DE). El diámetro basal (DB), área basal $(\mathrm{AB})$, altura de tallo $(\mathrm{AT})$, diámetro de copa promedio (DC), área de copa (AC) y número de hojas $(\mathrm{NH})$ se midió en cada individuo. El AC se determinó como se indicó en Velasco-García et al. (2016). El área de copa total (ACt) y el área basal total $(\mathrm{ABt})$ en cada UM se obtuvo sumando los valores individuales; éstos y DE se extrapolaron para una ha, y el promedio se obtuvo para cada población.

Cinco mil re-muestreos bootstrap de DE, ABt y ACt se realizaron en cada población. Los supuestos de normalidad y homogeneidad de varianzas se verificaron con la prueba de Shapiro-Wilks y de Levene, respectivamente, para todas las variables $(\mathrm{DB}, \mathrm{AB}, \mathrm{AT}, \mathrm{DC}$, $\mathrm{AC}, \mathrm{NH}, \mathrm{DE}, \mathrm{ABt}$ y $\mathrm{CC}$ ) usando el programa estadístico InfoStat (Di Rienzo et al., 2015). Ninguna variable cumplió con ambos supuestos, por tanto, las diferencias entre poblaciones se determinaron aplicando pruebas no paramétricas de varianza y comparaciones 
múltiples de rangos de Kruskal-Wallis (Kruskal \& Wallis, 1952; Conover \& Iman, 1981). La semejanza dendrométrica de las poblaciones se determinó usando el componente principal (CPrin1) de los valores dendrométricos absolutos (DB, AB, AT, DC, AC, NH) y con la técnica de conglomerados con base en la distancia euclidiana al cuadrado y el método de agrupación de promedio ponderado (Sokal \& Michener, 1958; McQuitty, 1966; NúñezColín \& Escobedo-López, 2011). Estos análisis se realizaron con el programa estadístico SAS (SAS Institute, 2002).

Estructura de estadios en las poblaciones: Los individuos de D. holmgrenii se clasificaron en reproductivos y no reproductivos, y en estadios de desarrollo de acuerdo a Velasco-García et al. (2016): plántula, juvenil 1 , juvenil 2, pre-reproductivo, adulto $1(0.11 \mathrm{a}$ $1.0 \mathrm{~m})$, adulto $2(1.01 \mathrm{a} 2.0 \mathrm{~m})$, adulto 3 (2.01 a $3.0 \mathrm{~m})$ y adulto $4(>3.0 \mathrm{~m})$. Las curvas de estructura poblacional se elaboraron con la densidad relativa de los estadios de desarrollo, y las diferencias se determinaron con la prueba de chi-cuadrada. Adicionalmente, las curvas de estructura poblacional se agruparon de acuerdo a los tipos estructurales típicos (Bongers, Popma, Meave-Del Castillo, \& Carabias, 1988; Peters, 1994; Martínez-Ramos \& Álvarez-Buylla, 1995).

Bongers et al. (1988) definió tres tipos estructurales; el tipo I tiene frecuencia alta de individuos en la primera o segunda clase de diámetro y con disminución gradual en clases superiores; el tipo II, tiene frecuencia alta de individuos en la primera clase diamétrica, en la segunda o tercer clase mal representados, aumento de la frecuencia en clases intermedias y disminución en las clases superiores; el tipo III, en la primera clase de diámetro con $50 \%$ o más individuos y con frecuencia muy baja y uniforme en clases siguientes. Peters (1994) también definió tres tipos estructurales, de los cuales el tipo I y II corresponde a los definidos por Bongers et al. (1988), mientras que el tipo III tiene individuos de aproximadamente del mismo tamaño, con ausencia en las primeras y últimas clases de diámetro. La estructura tipo I de Martínez-Ramos y Álvarez-Buylla (1995) corresponde al tipo I de Bogers et al. (1998), el tipo II presenta mayor frecuencia de individuos grandes que pequeños, mientas que el tipo III los individuos de las primeras y últimas clases diamétricas tienen frecuencia alta, con frecuencia baja en clases intermedias.

Los valores relativos de los parámetros estructurales (densidad, dominancia, frecuencia, diámetro basal, altura y área de copa) se calcularon considerando a los estadios de desarrollo como elementos diferenciados de la población. Los valores relativos se estimaron con la relación del valor absoluto de cada estadio dividido por el valor absoluto de todos los estadios multiplicados por 100 (Curtis \& McIntosh, 1951; Corella-Justavino et al., 2001). El índice de dominancia estructural (IDE) fue el componente principal uno (CPrin1) de los valores relativos de los parámetros estructurales. Las diferencias entre estadios del IDE dentro de cada población se determinaron con pruebas no paramétricas de varianza y comparaciones múltiples de rangos de Kruskal-Wallis (Kruskal \& Wallis, 1952; Conover \& Iman, 1981).

\section{Diversidad de estadios en las pobla-} ciones: La heterogeneidad (Peet, 1974) se determinó con el índice de Shannon $\mathrm{H}^{\prime}\left(\mathrm{H}^{\prime}=\right.$ -Lpilnpi) y la uniformidad E $\left(\mathrm{E}=\mathrm{H}^{\prime} / \operatorname{lnS}\right)$ de acuerdo a Magurran $(1988,2004)$ en cada unidad de muestreo, pi fue la proporción de individuos del estadio $i$, ln fue el logaritmo natural y $\mathrm{S}$ fue el número total de estadios.

Cinco mil remuestreos bootstrap de la heterogeneidad y la uniformidad se obtuvieron en cada población. Las diferencias entre poblaciones de la heterogeneidad y la equidad se determinaron con pruebas no paramétricas de varianza y comparaciones múltiples de rangos de Kruskal-Wallis (Kruskal \& Wallis, 1952; Conover \& Iman, 1981). Asimismo, análisis de conglomerados con base en las distancias euclidianas al cuadrado utilizando el agrupamiento jerárquico promedio ponderado (Sokal \& Michener, 1958; McQuitty, 1966; NúñezColín \& Escobedo-López, 2011) se realizaron 
para cada índice. Cinco mil remuestreos bootstrap de la densidad de cada estadio se obtuvieron en cada población, con los cuales se determinó la semejanza de estadios mediante el coeficiente de Sørensen cuantitativo ISct (Moreno, 2001; Chao, Chazdon, Colwell, \& Shen, 2006): $I S c t=(2 \mathrm{pN} / \mathrm{aN}+\mathrm{bN})$, donde $\mathrm{pN}$ fue la sumatoria de la abundancia más baja de cada uno de los estadios compartidos en ambas poblaciones, aN fue el número total de individuos en la población $\mathrm{A}, \mathrm{bN}$ fue el número total de individuos en la población $\mathrm{B}$. $\mathrm{La}$ semejanza de estadios también se analizó por agrupación jerárquica del promedio ponderado y distancia euclidiana al cuadrado (Southwood \& Henderson, 2000; Moreno, 2001) de la densidad absoluta de los estadios. Las pruebas no paramétricas y comparaciones múltiples se realizaron con el programa estadístico SAS (SAS Institute, 2002); mientras que los remuestreos y los análisis multivariados se efectuaron con el programa estadístico InfoStat (Di Rienzo et al., 2015).

\section{RESULTADOS}

Atributos dendrométricos de las poblaciones: La densidad promedio en las poblaciones fue $589 \pm 259$ individuos.ha-1, de los cuales $249 \pm 138$ fueron reproductivos y $339 \pm 148$ no reproductivos. La densidad promedio entre poblaciones fue de 147.9 a 954.3 plantas.ha $^{-1}$ (Cuadro 1). El porcentaje promedio de plantas reproductivas varió de 18.8 a $58.0 \%$ y el porcentaje de plantas no reproductivas fue entre 42.0 y $81.2 \%$ (Fig. 1). La hipótesis de igualdad entre poblaciones se rechazó ( $\mathrm{p}<0.0002)$ para la densidad (DE), área basal total $(\mathrm{ABt})$, área de copa total $(\mathrm{ACt})$, atributos estructurales individuales (diámetro basal DB, área basal AB, altura de tallo AT, diámetro de copa DC, área de copa $\mathrm{AC}$ y número de hojas NH) y CPrin1 (Cuadro 1). El CPrin1 de los atributos individuales explicó el $92.79 \%$ de la varianza; el $\mathrm{DB}$ y $\mathrm{AB}$ contribuyeron con el valor mayor (0.413) para explicar la variabilidad, mientras que $\mathrm{NH}$ tuvo influencia menor (0.398). El CPrin1 y el dendrograma (Fig. 2) mostraron tres grupos con semejanza dendrométrica.

Estructura de estadios en las poblaciones: La hipótesis de igual estructura poblacional se rechazó. Las curvas de estructura poblacional fueron estadísticamente diferentes $(\mathrm{p}<0.0213)$ entre todas las poblaciones,

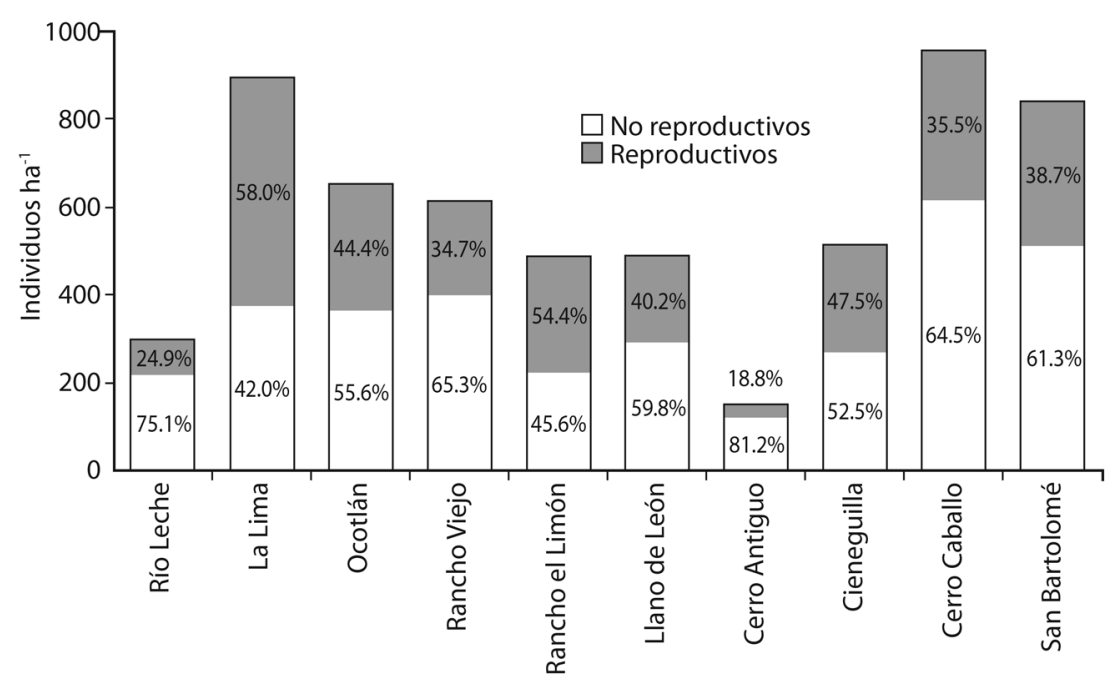

Fig. 1. Densidad de individuos de Dioon holmgrenii en diez poblaciones estudiadas en el sur de Oaxaca, México.

Fig. 1. Density of Individuals of Dioon holmgrenii in ten populations located in Southern Oaxaca, Mexico. 


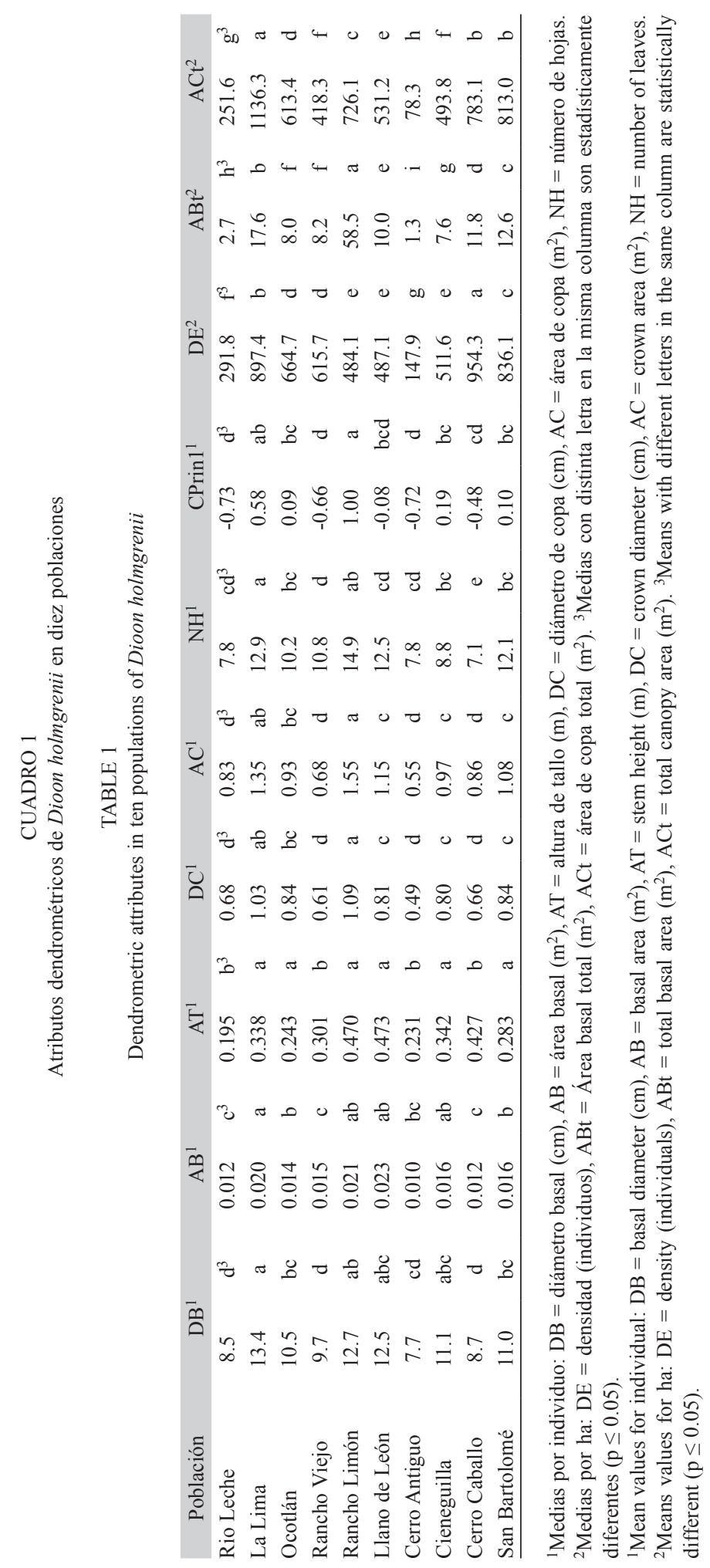




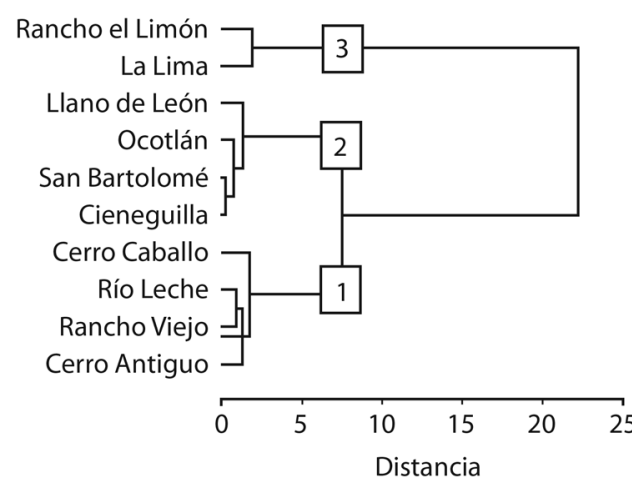

Fig. 2. Semejanza dendrométrica de diez poblaciones de Dioon holmgrenii. Los números arábigos (1, 2 y 3$)$ indican los grupos de poblaciones.

Fig. 2. Dendrometric similarity of ten populations of Dioon holmgrenii. The Arabic numerals (1,2, and 3 ) indicate the population groups.

excepto entre Cerro Caballo con Rancho Viejo $(\mathrm{p}=0.4618)$ y entre La Lima con Ocotlán $(\mathrm{p}=0.0931$, Anexo 1). Éstas curvas se agruparon en tres tipos estructurales y una variante de acuerdo a sus semejanzas con estructuras poblacionales típicas. La curva de estructura poblacional tipo I presentó $30 \%$ de plántulas y disminución casi constante en la proporción de individuos a estadios superiores (Fig. 3A). La curva de estructura poblacional tipo II presentó proporción alta de plántulas, disminución gradual en juvenil 1, juvenil 2 y pre-reproductivo, proporción alta de adulto $1 \mathrm{y}$ disminución gradual de adulto 2, adulto 3 y adulto 4 (Fig. 3B). Una variante de la estructura poblacional tipo II (tipo IIb) se caracterizó por proporción baja de plántulas, ligera disminución en juvenil 1 y juvenil 2, amento de pre-reproductivos, proporción muy alta de adulto 1 y disminución drástica en el resto de los estadios (Fig. 3C). Dos poblaciones presentaron una estructura poblacional diferente a las curvas de estructura poblacional típicas, la cual se denominó tipo $\mathrm{IV}$, se caracterizó por proporción baja en los estadios plántula, juvenil 2, pre-reproductivo, adulto 2, adulto $3 \mathrm{y}$ adulto 4 , y proporción alta de juvenil 1 y adulto 1 (Fig. 3D).

Diferencias del IDE $(p<0.0246)$ se encontraron entre estadios en todas las poblaciones, excepto en Río Leche $(p=0.2029)$ y en Cerro Antiguo $(\mathrm{p}=0.4743)$. Los estadios adulto $1 \mathrm{y}$ adulto 2 tuvieron los valores mayores de IDE en todas las poblaciones de $D$. holmgrenii; mientras que, los estadios pre-reproductivo, juvenil 1 y juvenil 2 mostraron IDE menores (Cuadro 2). Los primeros estadios de adulto tuvieron la mayor proporción de estróbilos femeninos ( $48 \%$ en adulto $1,33 \%$ en adulto 2 , $13 \%$ en adulto 3 y $6 \%$ en adulto 4 ) y masculinos (54 \% en adulto $1,38 \%$ en adulto $2,8 \%$ en adulto 3 y $0 \%$ en adulto 4 ) en las poblaciones de D. holmgrenii estudiadas. El CPrin1 de los valores relativos explicó el 77 \% de la varianza; el diámetro relativo tuvo influencia mayor (0.46) en explicar la viabilidad, mientras que la frecuencia relativa influyó menos $(0.34)$. El análisis multivariado mostró que el IDE agrupó a las poblaciones en tres grupos (Fig. 4A).

Diversidad de estadios en las poblaciones: La hipótesis de igualdad entre las poblaciones se rechazó para la diversidad de estadios. Todas las poblaciones fueron diferentes en heterogeneidad y uniformidad de estadios ( $p<0.0001)$, excepto La Lima con Llano de León para la heterogeneidad, Cieneguilla con Cerro Caballo $(p=0.1080)$ y Llano de León $(\mathrm{p}=0.1775)$ para la uniformidad. La heterogeneidad fue mayor en la población Cerro Caballo y menor en Río Leche, mientras que, la uniformidad fue mayor en Cerro Antiguo y menor en Ocotlán $(\mathrm{p}<0.0001$; Cuadro 3$)$. La semejanza de estadios registró los valores menores entre la población Cerro Antiguo con las de Cerro Caballo (ISct $=0.27$ ) y La Lima (ISct $=0.28)$, así como los mayores entre las poblaciones Cieneguilla con Llano de León $($ ISct $=0.88)$ y San Bartolomé con Ocotlán $($ ISct $=0.87)($ Anexo 2). La distancia euclidiana al cuadrado promedio entre las poblaciones fue de 16.0, Cerro Antiguo con Río Leche fueron los de mayor semejanza, mientras que Cerro Caballo con Río Leche las de semejanza menor (Anexo 2). El análisis multivariado definió a las poblaciones en tres grupos para la heterogeneidad, dos para uniformidad y cuatro para semejanza (Fig. 4B, Fig. 4C y Fig. 4D). 
CUADRO 3

Heterogeneidad $\left(\mathrm{H}^{\prime}\right)$ y uniformidad $(\mathrm{E})$ de estadios en diez poblaciones de Dioon holmgrenii

TABLE 3

Heterogeneity $\left(\mathrm{H}^{\prime}\right)$ and eveness $(\mathrm{E})$ of life stages in ten populations of Dioon holmgrenii

\begin{tabular}{lcccc}
\multicolumn{1}{c}{ Población } & \multicolumn{2}{c}{$\mathrm{H}^{\prime 1}$} & \multicolumn{2}{c}{$\mathrm{E}^{1}$} \\
Rio Leche & 1.053 & $\mathrm{i}$ & 0.799 & $\mathrm{f}$ \\
La Lima & 1.347 & $\mathrm{f}$ & 0.723 & $\mathrm{~g}$ \\
Ocotlán & 1.088 & $\mathrm{~h}$ & 0.575 & $\mathrm{i}$ \\
Rancho Viejo & 1.217 & $\mathrm{~g}$ & 0.680 & $\mathrm{~h}$ \\
Rancho Limón & 1.627 & $\mathrm{~b}$ & 0.825 & $\mathrm{c}$ \\
Llano de León & 1.362 & $\mathrm{f}$ & 0.805 & $\mathrm{e}$ \\
Cerro Antiguo & 1.437 & $\mathrm{e}$ & 0.878 & $\mathrm{a}$ \\
Cieneguilla & 1.519 & $\mathrm{~d}$ & 0.818 & $\mathrm{de}$ \\
Cerro Caballo & 1.708 & $\mathrm{a}$ & 0.820 & $\mathrm{~d}$ \\
San Bartolomé & 1.600 & $\mathrm{c}$ & 0.837 & $\mathrm{~b}$ \\
\hline
\end{tabular}

${ }^{1}$ Medias con distinta letra en la misma columna son estadísticamente diferentes $(\mathrm{p} \leq 0.05)$.

${ }^{1}$ Means with different letters in the same column are statistically different $(\mathrm{p} \leq 0.05)$.

\section{DISCUSIÓN}

La densidad promedio de individuos en las poblaciones de Dioon holmgrenii fue superior a lo reportado para D. merolae De Luca, Sabato \& Vázq. Torres (116 a 167 ha $^{-1}$ ) en Oaxaca y Chiapas, México (Flores-Vázquez, Valverde, \& Lucas-González, 2012; Lázaro-Zermeño, González-Espinoza, Mendoza, Martínez-Ramos, \& Quintana-Ascencio, 2011) y para D. purpusii Rose $\left(37 \mathrm{ha}^{-1}\right)$ en Oaxaca (Yáñez-Espinosa \& Sosa-Sosa, 2007), pero menor a lo mencionado para D. edule Lindl. (2100 a $4633 \mathrm{ha}^{-1}$ ) en Veracruz (Octavio-Aguilar et al., 2008; Vovides, 1990). Estas diferencias pueden estar relacionadas con el estado de conservación del hábitat, como se encontró en Zamia melanorrhachis D.W. Stev. y D. holmgrenii donde las perturbaciones influyeron negativamente en la calidad del mismo (López-Gallego, 2008; Velasco-García et al., 2016). Igualmente, las densidades mayores de Ceratozamia mirandae Vovides, Pérez-Farr. \& Iglesias y C. fuscoviridis Moore se encontraron en bosques 

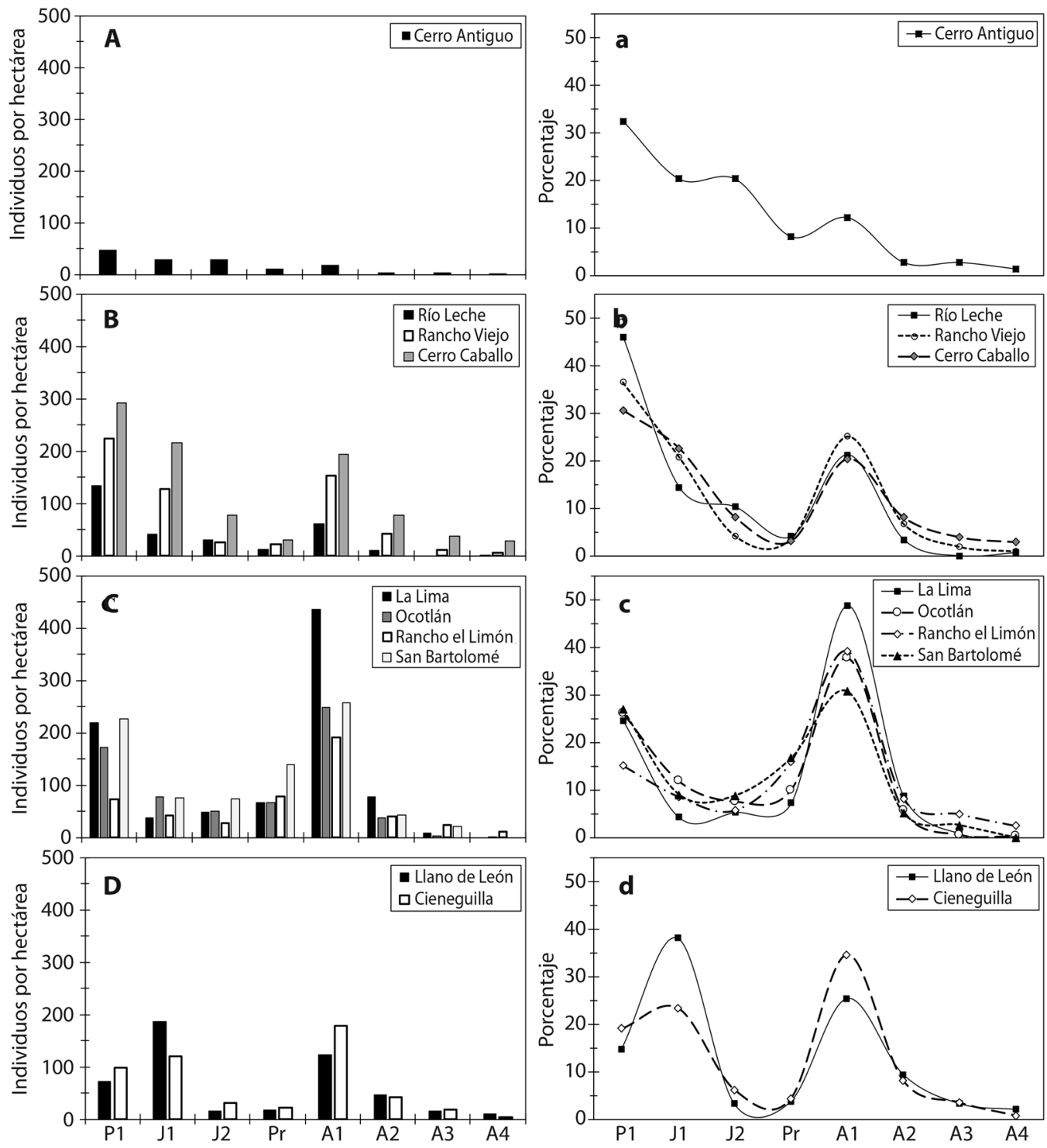

Fig. 3. Densidad promedio $(\mathbf{A}, \mathbf{B}, \mathbf{C}, \mathbf{D})$ por estadios $(\mathrm{Pl}=$ plántula, $\mathrm{J} 1=$ juvenil $1, \mathrm{~J} 2=$ juvenil 2 , $\operatorname{Pr}=$ prereproductivos, $\mathrm{A} 1=$ adulto $1, \mathrm{~A} 2=$ adulto $2, \mathrm{~A} 3=$ adulto $3, \mathrm{~A} 4=$ adulto 4$)$ y curva de estructura poblacional $(\mathbf{a}, \mathbf{b}, \mathbf{c}, \mathbf{d})$ de diez poblaciones de Dioon holmgrenii.

Fig. 3. Average density (A, B, C, D) of each life stage ( $\mathrm{P} 1=$ seedling, $\mathrm{J} 1=$ Youth $1, \mathrm{~J} 2=$ Youth $2, \mathrm{Pr}=$ pre-reproductive, $\mathrm{A} 1=$ adult $1, \mathrm{~A} 2=$ adult $2, \mathrm{~A} 3=$ adult $3, \mathrm{~A} 4=$ adult 4$)$ and population structure curve $(\mathbf{a}, \mathbf{b}, \mathbf{c}, \mathbf{d})$ for ten populations of Dioon holmgrenii.

conservados en comparación con aquellos perturbados (Pérez-Farrera et al., 2006; Pulido, Vargas-Zenteno, Vite, \& Vovides, 2015).

El porcentaje de individuos no reproductivos fue más variable entre las poblaciones evaluadas en este estudio que lo reportado para la misma especie (60.6 y $72.5 \%)$ entre sitios sin cambio de uso de suelo (Velasco-García et al., 2016) y para $D$. edule (78.4 a $91.7 \%$ ) en condiciones similares (Octavio-Aguilar et al., 

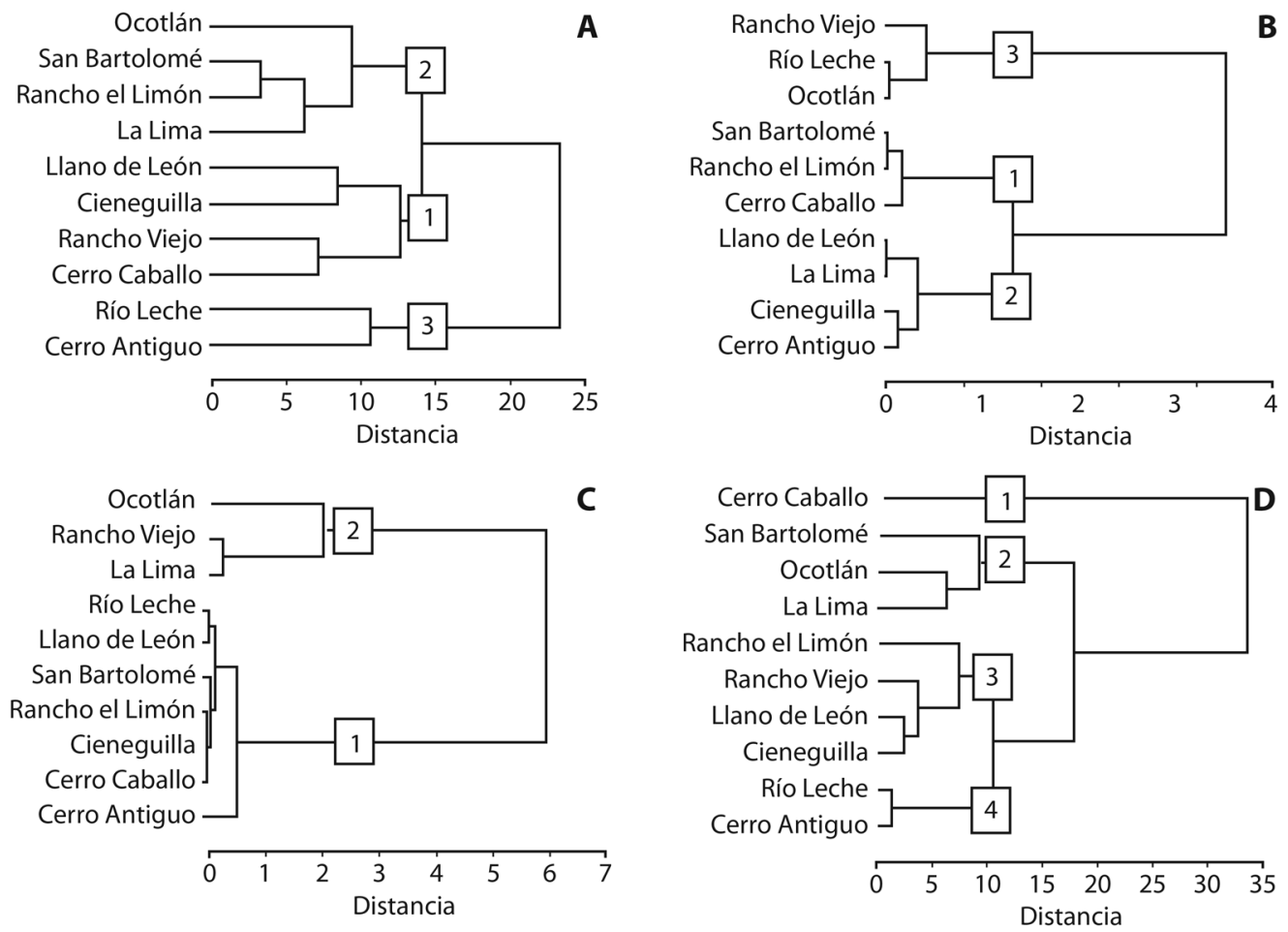

Fig. 4. Dendrogramas de semejanza de diez poblaciones de Dioon holmgrenii. Semejanza: A = dominancia estructural, $\mathbf{B}=$ heterogeneidad, $\mathbf{C}=$ uniformidad y $\mathbf{D}=$ de estadios. Los números arábigos (1, 2, 3 y 4 ) indican los grupos de poblaciones en cada dendrograma.

Fig. 4. Similarity dendrograms of ten populations of Dioon holmgrenii. Similarity: $\mathbf{A}=$ structural dominance, $\mathbf{B}=$ heterogeneity, $\mathbf{C}=$ evenness, and $\mathbf{D}=$ of stages. The Arabic numerals $(1,2,3$, and 4$)$ indicate the population groups in each dendrogram.

2008). Aunque la densidad, área basal total y área de copa total fueron las variables menores en las poblaciones Río Leche y Cerro Antiguo del presente estudio, el porcentaje de individuos no reproductivos fue mayor que en Cerro Caballo, debido a que la agricultura y ganadería se suspendieron en las dos primeras, con lo que aumentó la germinación por una incidencia mayor de luz y disponibilidad de suelo que mejora sus propiedades químicas y capacidad de captación de agua (Cruz-Ruiz et al., 2012).

Las poblaciones con valores dendrométricos individuales $(\mathrm{DB}, \mathrm{AB}, \mathrm{AT}, \mathrm{DC}, \mathrm{AC}$, $\mathrm{NH}$ ) bajos, intermedios y altos fueron clasificados en tres grupos dendrométricos distintos por el análisis multivariado de componentes principales (CPrin1) y de conglomerados. Lo anterior porque los porcentajes de individuos reproductivos y no reproductivos tuvieron asociación fuerte con los valores dendrométricos individuales. El coeficiente de correlación de Pearson fue positivo entre los valores dendrométricos (excepto AT) con el porcentaje de individuos reproductivos $(\mathrm{r}=0.71$ a $0.92, \mathrm{p}<$ 0.022 ) y negativo entre éstos con el porcentaje de individuos no reproductivos $(\mathrm{r}=-0.71$ a -0.92, p < 0.022). Asimismo, la correlación entre el CPrin1 de los valores dendrométricos con la proporción de individuos reproductivos fue positiva $(r=0.91, p=0.0002)$ y de éstos con la proporción de individuos no reproductivos fue negativa $(r=-0.91, p=0.0002)$.

La curva de estructura poblacional tipo I que se obtuvo en el presente estudio sugiere 
una reproducción adecuada e incorporación continua a estadios superiores (Bongers et al., 1988; Pulido et al., 2015). Esta estructura correspondió a $D$. edule en sitios con baja perturbación humana (Vovides, 1990), Cycas circinalis L. en poblaciones sin aprovechamiento de hojas y semillas (Krishnamurthy et al., 2013), Cycas micronesica K. D. Hill en localidades sin ataque de la escama de las cícadas Aulacaspis yasumatsui Takagi (Marler \& Lawrence, 2012), Ceratozamia mexicana Brongn en sitios con barrancas y laderas pronunciadas (AlejandreRosas, Sánchez-Tinoco, \& Vázquez-Torres, 1990), Zamia debilis L.f(Negrón-Ortiz \& Breckon, 1989), C. matudae Lundell (Pérez-Farrera \& Vovides, 2004) y Zamia melanorrhachis (López-Gallego, 2008) en bosques conservados, remanentes y secundarios. La estructura poblacional tipo I es característica de especies tolerantes a la sombra (Peters, 1994; MartínezRamos \& Álvarez-Buylla, 1995). La tolerancia a la sombra no se conoce en D. holmgrenii, pero puede tener comportamiento similar a otras especies del género Dioon; por ejemplo, las plántulas de $D$. sonorense (De Luca, Sabato et Vázq. Torres) Chemnick, T.J. Greg. et Salas-Mor. tienen mayor tolerancia a la sombra que los estadios superiores (Álvarez-Yepiz, Búrquez, \& Dovčiak, 2014). La frecuencia alta de individuos en estadios inferiores puede indicar abundancia de sitios favorables para el crecimiento inicial en estructuras poblacionales tipo I (Martínez-Ramos \& Álvarez-Buylla, 1995). La suspensión de la agricultura en algunos sitios mejoró la captación de agua y las propiedades químicas del suelo, generando condiciones favorables para el éxito de los primeros estadios de D. holmgrenii (Cruz-Ruiz et al., 2012). En la población Cerro Antiguo, D. holmgrenii crece en suelos rocosos, en los cuales posiblemente se generaron micrositios favorables para competir, al igual que lo encontrado en $D$. sonorense, donde todos sus estadios se asociaron positivamente con las rocas, creando micrositios de bajo nivel de competencia interespecífica (Álvarez-Yepiz et al., 2014).

La curva de estructura poblacional tipo II que se obtuvo en el presente estudio, indica reproducción adecuada con establecimiento esporádico de plántulas e interrupción temporal de la repoblación por daños directos a la misma (Bongers, 1988; Peters, 1994). Esta estructura poblacional es típica en sitios de $D$. holmgrenii sin deforestación y poco perturbados, como el caso de la población Cerro Caballo (VelascoGarcía et al., 2016), y se ha registrado en Ceratozamia mirandae y Cycas circinalis en sitios sin disturbios humanos (Pérez-Farrera et al., 2006; Varghese, Krishnamurthy, \& Ticktin, 2012). La proporción alta de plántulas y disminución de juveniles son resultado de alteraciones por incendios en los últimos años; los incendios, dependiendo de su intensidad, pueden estimular la producción de semillas de las cícadas, pero también provocar alta mortalidad de plántulas que pasarían al siguiente estadio (Ornduff, 1991; Negrón-Ortiz \& Gorchof, 2000; Asmussen, 2009; Pérez-Farrera et al., 2006). Deforestaciones no se encontraron en Cerro Caballo, pero sí indicios de ocurrencia de incendios en años anteriores (Velasco-García et al., 2016).

La estructura poblacional tipo IIb que se encontró en este estudio sugiere limitantes ambientales drásticas para el establecimiento y supervivencia de individuos en los primeros estadios (Martínez-Ramos \& Álvarez-Buylla, 1995). De acuerdo con Velasco-García et al. (2016) este tipo de estructura fue característico de $D$. holmgrenii en sitios con incidencia alta de agricultura y ganadería. Estructuras poblacionales similares a este tipo se encontraron para $D$. merolae en sitios donde las hojas fueron cosechadas y en poblaciones con producción baja de semillas (Lázaro-Zermeño et al., 2011; Flores-Vázquez et al., 2012), así como debido a alteraciones en otras cícadas como Zamia melanorrhachis, Encephalartos cycadifolius (Jacq.) Lehm., y Cycas micronesica (Raimondo \& Donaldson, 2003; López-Gallego, 2008; Marler, 2012).

Las curvas de estructura poblacional tipo IV que mostraron dos poblaciones de este estudio parecen ser típicas de poblaciones de cícadas con restricciones bióticas y abióticas para la producción de semillas; por ejemplo, 
estructuras similares se reportaron en poblaciones de Encephalartos villosus Lem. donde una proporción alta de semillas fue dañada por el picudo de cícadas Antliarhinus zamiae (Thunberg) y en poblaciones de Cycas circinalis con una cosecha intensa de hojas y semillas (Raimondo \& Donaldson, 2003; Krishnamurthy et al., 2013). La producción baja de semillas de $D$. holmgrenii en estas poblaciones pudo deberse a la decapitación de plantas reproductivas en zonas de agricultura de la población Cieneguilla y en zonas de ganadería intensiva en la población Llano de León (Velasco-García et al., 2016), al igual que lo encontrado para $D$. edule que tuvo una menor tasa de crecimiento poblacional con plantas reproductivas decapitadas en comparación con aquellas sin decapitación (Octavio-Aguilar et al., 2008). La estructura poblacional tipo IV también representó limitantes para el establecimiento y supervivencia, como en el caso de Cycas micronesica con mortalidad alta de estadios inferiores causado por Aulacaspis yasumatsui (Marler \& Lawrence, 2012). La relación entre D. holmgrenii con otros organismos no se investigó en este trabajo; sin embargo, larvas de Eumaeus childrenae (Gray) se observaron alimentándose de las hojas nuevas de $D$. holmgrenii en todas las poblaciones (Velasco-García et al., 2016). Las larvas pueden afectar la reincorporación de plantas y la estructura poblacional como fue el caso de la relación entre Eumaeus devora Hubner. y Ceratozomia matudae (Pérez-Farrera \& Vovides, 2004). Asimismo, E. toxea Godart interactúa solo con Zamia paucijuga Wienland en algunas poblaciones de D. holmgrenii (Ruiz-García et al., 2015), similar a lo que ocurre con E. childrenae en poblaciones donde Zamia fischeri Miq. y Z. loddigesii Miq. crecen juntas (Contreras-Medina, Ruiz-Jiménez, \& Luna-Vega, 2003).

El índice de valor de importancia (IVI) jerarquiza la dominancia de especies arbóreas en rodales mezclados (Curtis \& McIntosh, 1951; García-Mayoral, Valdez-Hernández, LunaCavazos, \& López-Morgado, 2015), mientras que el índice de valor forestal (IVF) evalúa la estructura bidimensional de la vegetación, considerando tanto el plano vertical como horizontal (Corella-Justavino et al., 2001; ZarcoEspinosa, Valdez-Hernández, Ángeles-Pérez, \& Castillo-Acosta, 2010). Ambos índices se usaron para evaluar la importancia estructural de estadios de D. holmgrenii (Velasco-García et al., 2016); sin embargo, y debido a que cada índice jerarquiza de manera diferente a la mayoría de las especies o estadios (ZarcoEspinosa et al., 2010; López-Toledo, ValdezHernández, Pérez-Farrera, \& Cetina-Alcalá, 2012; Carreón-Santos \& Valdez-Hernández, 2014; Velasco-García et al., 2016), en el presente estudio se calculó el índice de dominancia estructural (IDE) que sintetiza tanto los elementos del IVI como del IVF. Los estadios con IDE altos tuvieron valores altos de densidad, área basal, frecuencia, diámetro basal, altura y área de copa, en contraste con los estadios con valores bajos de estos atributos. Los IDE altos de adulto $1 \mathrm{y}$ adulto 2 pueden favorecer la permanencia de las poblaciones si se moderan las actividades agrícolas y el pastoreo intensivo, debido a que estos estadios poseen mayor vigor reproductivo que los otros (Lázaro-Zermeño et al., 2011; Velasco-García et al., 2016). El grupo 1 de semejanza por IDE lo integraron las poblaciones con los menores valores de IDE para el estadio pre-reproductivo, el grupo 2 lo integraron poblaciones con el mismo tipo estructural y con valores positivos de IDE para los estadios adultos 1 y 2 , mientras que los estadios de las poblaciones del grupo 3 no presentaron diferencias en IDE.

El primer grupo de heterogeneidad de estadio se integró con poblaciones con valores altos de $\mathrm{H}^{\prime}$. El segundo se formó con poblaciones con valores intermedios, y el tercero lo constituyeron poblaciones con valores bajos. El grupo 1 tuvo valores iguales o mayores a los sitios sin cambio de uso de suelo y baja perturbación $\left(\mathrm{H}^{\prime}=1.6\right)$, el grupo 2 registró valores similares a los sitios con agricultura $\left(\mathrm{H}^{\prime}=1.35\right)$ y sitios en recuperación $\left(H^{\prime}=1.48\right)$, mientras que el grupo 3 mostró valores menores a los sitios con ganadería intensiva $\left(\mathrm{H}^{\prime}=1.31\right)$ con base en los valores de $\mathrm{H}^{\prime}$ reportados para D. holmgrenii (Velasco-García et al., 2016). El grupo 1 
de uniformidad (E) tuvo valores altos de $\mathrm{E} y$ similares a los sitios sin cambio de uso de suelo reportados para $D$. holmgrenii, mientras que el grupo 2 registró valores bajos e inferiores a los reportados para sitios con cambio de uso de suelo (Velasco-García et al., 2016). Los valores altos de $\mathrm{H}^{\prime}$ y $\mathrm{E}$ de estadios son característicos de poblaciones con poca perturbación (Velasco-García et al., 2016); sin embargo, los valores altos de $\mathrm{H}^{\prime}$ y $\mathrm{E}$ en sitios con agricultura o ganadería intensiva pueden deberse a que los propietarios no dañan intencionalmente las plantas de D. holmgrenii. Velasco-García et al. (2016) encontraron que los sitios con cambio de uso de suelo de las poblaciones Rancho el Limón y San Bartolomé presentaron mayor densidad de individuos que en sitios sin cambio de uso de suelo de poblaciones con incidencia alta de incendios forestales. Esto sugiere que esta especie puede ser conservada en poblaciones o sitios con prácticas de agricultura o ganadería moderadas, donde se garanticen los procesos de reproducción y reincorporación de las plantas de esta especie.

Los valores del coeficiente de Sorensen cuantitativo en la mitad de las comparaciones entre poblaciones ( 22 de 45$)$ indicaron que al menos cuatro estadios fueron diferentes entre éstas (ISct $<0.68$ ). Esto apoyó el resultado obtenido por el índice de diversidad $\left(\mathrm{H}^{\prime}\right)$ y uniformidad (E), donde la mayoría de las poblaciones fueron diferentes. Los bajos valores de semejanza pueden estar relacionados con las diferencias en la historia de perturbaciones ocurridas en las poblaciones (Velasco-García et al., 2016). El dendrograma de semejanza de estadios separó a la población Cerro Caballo de las otras poblaciones. Estas últimas se agruparon por el tipo de actividad antropógena existente en áreas deforestadas, lo cual indicó una semejanza mayor entre poblaciones con agricultura, ganadería intensiva y sitios en recuperación. Velasco-García et al. (2016) encontraron mayor semejanza de estadios entre sitios con perturbaciones similares.

\section{AGRADECIMIENTOS}

A las autoridades (comunales, ejidales y administrativas), poseedores de los terrenos y guías de campo por permitir y apoyar en la toma de datos. A Guillermo Sánchez De la Vega, Noé Ruiz García, Ignacio Mejía Cuevas y Carlos A. Ruiz Jiménez por el apoyo para la ubicación de las poblaciones. A Silvestre B. Ramírez López, Juan Luis Hernández Hernández, Eduardo Molina García y Edgar López Velasco por apoyar en la toma de datos. Al CONACyT por la beca otorgada al primer autor para realizar estudios de doctorado en el Colegio de Postgraduados.

\section{RESUMEN}

Dioon holmgrenii esta catalogada en peligro de extinción y es endémica del sur de Oaxaca, México. A pesar de la situación de esta especie, no existe información específica sobre aspectos ecológicos para cada población. Esto dificulta la autorización de planes de manejo para su conservación. El objetivo de este trabajo fue determinar los atributos dendrométricos, la estructura poblacional y la diversidad de estadios en diez poblaciones de D. holmgrenii localizadas en la Sierra Madre del Sur de Oaxaca. Cuatro unidades de muestreo (UM) de 25 x $50 \mathrm{~m}$ cada una $\left(1250 \mathrm{~m}^{2}\right)$ se establecieron por población, $40 \mathrm{UM}$ en total $\left(50000 \mathrm{~m}^{2}\right)$. La densidad de plantas, el diámetro basal (DB), área basal (AB), altura de tallo (AT), diámetro de copa (DC), área de copa (AC) y número de hojas (NH) de los individuos se evaluó de enero a mayo 2013. Con estos datos se determinó la estructura poblacional, índice de dominancia estructural (IDE), heterogeneidad ( $\left.\mathrm{H}^{\prime}\right)$, uniformidad (E) y semejanza (ISct) de estadios. La densidad en las poblaciones varió de 148 a 954 plantas ha ${ }^{-1}$. El área basal total fluctuó de 1.3 a $17.6 \mathrm{~m}^{2} \mathrm{ha}^{-1} \mathrm{y}$ área de copa total osciló de 78.3 a $1136.3 \mathrm{~m}^{2} \mathrm{ha}^{-1}$. Los atributos dendrométricos de los individuos $(\mathrm{DB}=7.7$ a $13.4 \mathrm{~cm}, \mathrm{AB}=0.010$ a $0.023 \mathrm{~m}^{2}, \mathrm{AT}=0.20$ a $0.47 \mathrm{~m}, \mathrm{DC}=0.49$ a $1.09 \mathrm{~m}, \mathrm{AC}$ $=0.55$ a $1.55 \mathrm{~m}^{2}$ y NH $=7.06$ a 14.90) fueron diferentes entre las poblaciones $(\mathrm{p}<0.05)$. Adulto 1 y adulto 2 tuvieron mayor IDE $(0.01$ a 5.63$)$ en todas las poblaciones. La estructura poblacional más común fue aquella donde juvenil 1 y juvenil 2 tuvieron las proporciones más bajas de individuos, mientras que adulto 1 tuvo la más alta. La heterogeneidad $\left(\mathrm{H}^{\prime}=1.05\right.$ a 1.71) y uniformidad $(\mathrm{E}=0.57 \mathrm{a}$ 0.88 ) de estadios fueron significativamente diferentes entre las poblaciones $(\mathrm{p}<0.05)$, con al menos cuatro estadios 
$(3.91=48.9 \times 8 / 100)$ diferentes entre ellas para la mitad de las comparaciones (22 de 45) de semejanza (ISct < 0.68). La diversidad alfa de estadios fue alta en poblaciones donde no se dañaron las plantas, en contraste a la diversidad beta que fue baja entre poblaciones con presencia de actividad humana. Estadios con alto vigor reproductivo (adulto 1 y adulto 2) presentaron una mayor dominancia estructural en todas las poblaciones. Estos resultados pueden ayudar a la conservación in-situ de D. holmgrenii y se recomienda sean considerados en la elaboración y aplicación de planes de manejo de sus poblaciones.

Palabras clave: área basal y de copa, densidad, dominancia estructural, heterogeneidad, uniformidad, semejanza de estadios.

\section{REFERENCIAS}

Alejandre-Rosas, J. A., Sánchez-Tinoco, M. Y., \& Vázquez-Torres, M. (1990). Estructura poblacional de Ceratozamia mexicana Brongn. (Zamiaceae) en un bosque del centro de Veracruz. La Ciencia y El Hombre, 5, 93-112.

Álvarez-Yepiz, J. C., Búrquez, A., \& Dovčiak, M. (2014). Ontogenetic shifts in plant-plant interactions in a rare cycad within angiosperm communities. Oecologia, $175,725-735$.

Asmussen, B. (2009). Another burning question: HunterGatherer exploitation of Macrozamia spp. Archaeology in Oceania, 44, 142-149.

Bongers, F., Popma, J., Meave-Del Castillo, J., \& Carabias, J. (1988). Structure and floristic composition of the lowland rain forest of Los Tuxtlas, Mexico. Vegetatio, $74,55-80$.

Carreón-Santos, R. J., \& Valdez-Hernández, J. I. (2014). Estructura y diversidad arbórea de la vegetación secundaria derivada de una selva mediana subperennifolia en Quintana Roo. Revista Chapingo Serie Ciencias Forestales y del Ambiente, 20, 119-130.

Chao, A., Chazdon, R. L., Colwell, R. K., \& Shen, T-J. (2006). Abundance-based similarity indices and their estimation when there are unseen species in samples. Biometrics, 62, 361-371.

Chemnick, J., Gregory, T., \& Morales, S. (2010). Dioon holmgrenii. In The IUCN Red List of Threatened Species 2010: e.T42129A10660365. Recuperate of http://dx.doi.org/10.2305/IUCN.UK.2010-3.RLTS. T42129A10660365.en

Christenhusz, M. J. M., Reveal, J. L., Farjon, A., Gardner, M. F., Mill, R., \& Chase, M. W. (2011). A new classification and linear sequence of extant gymnosperms. Phytotaxa, 19, 55-70.
Condamine, F. L., Nagalingum, N. S., Marshall, C., \& Morlon, H. (2015). Origin and diversification of living cycads: a cautionary tale on the impact of the branching process prior in Bayesian molecular dating. BMC Evolutionary Biology, 15, 65 .

Conover, W. J., \& Iman, R. L. (1981). Rank transformation as a bridge between parametric and nonparametric statistics. The American Statistician, 35, 124-129.

Contreras-Medina, R., Ruiz-Jiménez, C. A., \& LunaVega, I. 2003. Caterpillars of Eumaeus childrenae (Lepidoptera: Lycaenidae) feeding on two species of cycads (Zamiaceae) in the Huasteca region, Mexico. Revista de Biología Tropical, 51, 201-204.

Corella-Justavino, F., Valdez-Hernández, J. I., Cetina-Alcalá, V. M., González-Cossio, F. V., Trinidad-Santos, A., \& Aguirre-Rivera, J. R. 2001. Estructura forestal de un bosque de mangles en el noreste del estado de Tabasco, México. Revista Ciencia Forestal en México, 26, 73-102.

Cruz-Ruiz, E., Cruz-Ruiz, A., Aguilera-Gómez, L. I., Norman-Mondragón, H. T., Velázquez, R. A., NavaBernal, G., Dendooven, L., \& Reyes-Reyes, B. G. (2012). Efecto en las características edáficas en un bosque templado por el cambio de uso de suelo. Terra Latinomericana, 30, 189-197.

Curtis, J. T., \& McIntosh, R. P. (1951). An upland forest continuum in the prairie-forest border region of Wisconsin. Ecology, 32, 476-496.

Dawson, J. W. (1883). On the Cretaceous and Tertiary floras of British Columbia and the Northwest Territory. Transactions of the Royal Society of Canada, 1, 15-34.

Di Rienzo, J. A., Casanoves, F., Balzarini, M. G., Gonzalez, L., Tablada, M., \& Robledo, C. W. (2015). InfoStat versión 2015. Córdoba, Argentina: Grupo InfoStat, FCA, Universidad Nacional de Córdoba.

Flores-Vázquez, J. C., Valverde, T., \& Lucas-González, J. L. (2012). How critical are the early life-cycles stages in the population ecology of cycads? A case study with Dioon merolae in southeastern Mexico. Memoirs of the New York Botanical Garden, $106,40-58$

García-Mayoral, L. E., Valdez-Hernández, J. I., LunaCavazos, M., \& López-Morgado, R. (2015). Estructura y diversidad arbórea en sistemas agroforestales de café en la Sierra de Atoyac, Veracruz. Madera y Bosques, 21, 69-82.

Krishnamurthy, V., Mandle, L., Ticktin, T., Ganesan, R., Sannesh, C. S., \& Varghese, A. 2013. Conservation status and effects of harvest on an endemic multipurpose cycad, Cycas circinalis L., Western Ghats, India. Tropical Ecology, 54, 309-320. 
Kruskal, W. H., \& Wallis, W. A. 1952. Use of ranks in onecriterion variance analysis. Journal of the American Statistical Association, 47, 583-621.

Lázaro-Zermeño, J. M., González-Espinoza, M., Mendoza, A., \& Martínez-Ramos, M. (2012). Historia natural de Dioon merolae (Zamiaceae) en Chiapas, México. Botanical Sciences, 90, 73-87.

Lázaro-Zermeño, J. M., González-Espinoza, M., Mendoza, A., Martínez-Ramos, M., \& Quintana-Ascencio, P. F. (2011). Individual growth, reproduction and population dynamics of Dioon merolae (Zamiaceae) under different leaf harvest histories in central Chiapas, Mexico. Forest Ecology and Management, 261, 427-439.

López-Gallego, C. 2008. Demographic variation in cycad populations inhabiting contrasting forest fragments. Biodiversity and Conservation, 17, 1213-1225.

López-Toledo, J. F., Valdez-Hernández, J. I., Pérez-Farrera M. A., \& Cetina-Alcalá, V. M. (2012). Composición y estructura arbórea de un bosque tropical estacionalmente seco en la Reserva de la Biosfera La Sepultura, Chiapas. Revista Mexicana de Ciencias Forestales, 3, 44-56.

McQuitty, L. L. (1966). Similarity analysis by reciprocal pairs for discrete and continuous data. Educational and Psychological Measurements, 26, 825-831.

Magurran, A. E. (1988). Ecological diversity and its measurement. Princeton, USA: Princeton University Press.

Magurran A. E. (2004). Measuring biological biodiversity. Malden, USA: Blackwell Publishing.

Mamay, S. H. (1976). Paleozoic origin of the cycads. Professional Paper 934. Washington, USA: Geological Survey.

Marler, T. E. (2012). Cycad Aulacaspis scale invades the Mariana Islands. Memoirs of the New York Botanical Garden, 106, 20-35.

Marler, E. M., \& Lawrence, J. H. (2012). Demography of Cycas micronesica on Guam following introduction of the armored scale Aulacaspis yasumatsui. Journal of Tropical Ecology, 28, 233-242.

Martínez-Ramos, M., \& Álvarez-Buylla, E. (1995). Ecología de poblaciones de plantas de una selva húmeda en México. Boletín de la Sociedad Botánica de México, 56, 121-153.

Moreno, C. E. (2001). Métodos para medir la biodiversidad. Zaragoza, España: CYTED, ORCYTUNESCO \& SEA.

Negrón-Ortiz, V., \& Breckon, G. J. (1989). Population structure in Zamia debilis (Zamiaceae). I. Size classes, leaf phenology, and leaf turnover. American Journal of Botany, 76, 891-900.
Negrón-Ortiz, V., \& Gorchof, D. L. (2000). Effects of fire season and postfire herbivory on the Cycad Zamia pumila (Zamiaceae) in slash pine savanna, Everglades National Park, Florida. International Journal of Plant Sciences, 161, 659-669.

Nicolalde-Morejón, F., González-Astorga, J., Vergara-Silva, F., Stevenson, D. W., Rojas-Soto, O., \& MedinaVillarreal, A. (2014). Biodiversidad de Zamiaceae en México. Revista Mexicana de Biodiversidad, $85, \mathrm{~S} 114-\mathrm{S} 125$.

Núñez-Colín, K., \& Escobedo-López, D. (2011). Uso correcto del análisis clúster en la caracterización de gemoplasma vegetal. Agronomía Mesoamericana, $22,415-427$.

Octavio-Aguilar, P., González-Astorga, J., \& Vovides, A. P. (2008). Population dynamics of the Mexican cycad Dioon edule Lindl. (Zamiaceae): life history stages and management impact. Botanical Journal of the Linnean Society, 157, 381-391.

Ornduff, R. (1991). Coning phenology of the cycad Macrozamia riedlei (Zamiaceae) over a five-year interval. Bulletin of the Torrey Botanical Club, 118, 6-11.

Ortíz-Pérez, M. A., Hernández-Santana, J. R., \& Figueroa, M. E. (2004). Reconocimiento fisiográfico y geomorfológico. In A. J. García-Mendoza, M. J. Ordoñez, \& M. Briones-Salas (Eds.), Biodiversidad de Oaxaca (pp. 43-54). México: Instituto de Biología UNAM-Fondo Oaxaqueño para la Conservación de la Naturaleza-World Wildlife Found.

Osborne, R., Calonje, M. A., Hill, K. D., Stanberg, L., \& Stevenson, D. W. (2012). The world list of cycads. Memoirs of the New York Botanical Gardens, 106, 480-510.

Peet, R. K. (1974). The measurements of species diversity. Annual Review of Ecology and Systematics, $5,285-307$

Pérez-Farrera, M. A, Vovides, A. P., Octavio-Aguilar, P., González-Astorga, J., De la Cruz-Rodríguez J., Hernández-Jonapá, R., \& Villalobos-Méndez, S. M. (2006). Demography of the cycad Ceratozamia mirandae (Zamiaceae) under disturbed and undisturbed conditions in a biosphere reserve of Mexico. Plant Ecology, 187, 97-108.

Pérez-Farrera, M. A., \& Vovides, A. P. (2004). Spatial distribution, population structure, and fecundity of Ceratozamia mirandae Lundell (Zamiaceae) in El Triunfo Biosfere Reserve, Chiapas, México. Botanical Review, 70, 299-311.

Pérez-Farrera, M. A., Quintana-Ascencio, P. F., \& Salvatierra-Izaba, B. (2000). Population dynamics of Ceratozamia matudai (Zamiacae) in El Triunfo Biosfere Reserve, Chiapas, México. Journal of the Torrey Botanical Society, 127, 291-299. 
Peters, C. M. (1994). Sustainable harvest of non-timber plant resources in tropical moist forest: an ecological primer. Washington, USA: Biodiversity Support Program.

Pulido, M. T., Vargas-Zenteno, M., Vite, A., \& Vovides, A. P. (2015). Range extension of the endangered Mexican cycad Ceratozamia fuscoviridis Moore (teosintle): implications for conservation. Tropical Conservation Science, 8, 778-795.

Raimondo, D. C., \& Donaldson, J. S. (2003). Responses of cycads with different life histories to the impact of plant collecting: simulation models to determine important life history stages and population recovery times. Biological Conservation, 111, 345-358.

Ruiz-García, N., Méndez-Pérez, B. Y., Velasco-García, M. V., Sánchez-de la Vega, G., \& Rivera-Nava, J. L. (2015). Ecología, distribución, ciclo biológico y tabla de vida de Eumaeus toxea (Lepidoptera: Lycaenidae) en la provincia fisiográfica Costa de Oaxaca, México. Revista Mexicana de Biodiversidad, 86, 998-1003.

SAS (Statistical Analysis System). (2002). SAS/STAT computer software. Release 9.00. Cary, USA: SAS Institute Inc.

SEMARNAT (Secretaría del Medio Ambiente y Recursos Naturales). (2010). Norma Oficial Mexicana NOM059-SEMARNAT-2010, Protección ambiental - Especies nativas de México de flora y fauna silvestres - Categorías de riesgo y especificaciones para su inclusión, exclusión o cambio - Lista de especies en riesgo. D.F., México: Diario Oficial de la Federación. 2da Sección, 30 de diciembre de 2010.

Sokal, R. R., \& Michener, C. D. (1958). A statistical methods for evaluating systematic relationships. University of Kansas Science Bulletin, 38, 1409-1438.

Southwood, T. R. E., \& Henderson, P. A. (2000). Ecological methods (3rd ed.). USA: Blackwell Science Ltd. Oxford.

Varghese, A., Krishnamurthy, V., \& Ticktin, T. (2012). Harvest, use, and ecology of Cycas circinalis L. a case study in the Nilgiri Biosphere Reserve area, Western Ghats, India. Memoirs of the New York Botanical Garden, 106, 178-191.

Velasco-García, M. V., Valdez-Hernández, J. I, RamírezHerrera, C., Hernández-Hernández, M. L., LópezUpton, J., López-Mata, L., \& López-Sánchez, H. 2016. Estructura, heterogeneidad de estadios y patrón de dispersión espacial de Dioon holmgrenii (Zamiaceae). Botanical Sciences, 94, 75-87.

Velasco-García, M. V., Sánchez-De la Vega, G., RuizGarcía, N., Ruiz-Jiménez, C., Méndez-Pérez, B. Y., \& Mejía-Cuevas, I. (2011). Distribución de Dioon holmgrenii (Zamiaceae) y su estado de conservación. En L. J. Toledo-Flores (Ed), $13^{\circ}$ Foro Estatal de Investigación Científica y Tecnológica (pp. 495-500). Oaxaca, México: Consejo Oaxaqueño de Ciencia y Tecnología.

Vovides, A. P. (1990). Spatial distribution, survival, and fecundity of Dioon edule (Zamiaceae) in a tropical deciduous forest in Veracruz, Mexico, with notes on its habitat. American Journal of Botany, $77,1532-1543$.

Vovides, A. P., \& Iglesias, C. G. (1994). An integrated conservation strategy for the cycad Dioon edule Lindl. Biodiversity and Conservation, 3, 137-141.

Yáñez-Espinosa, L., \& Sosa-Sosa, F. (2007). Population structure of Dioon purpusii Rose in Oaxaca, Mexico. Neotropical Biology and Conservation, 2, 46-54.

Zarco-Espinosa, V. M., Valdez-Hernández, J. I., ÁngelesPérez, G., \& Castillo-Acosta, O. (2010). Estructura y diversidad de la vegetación arbórea del parque estatal Agua Blanca, Macuspana, Tabasco. Universidad y Ciencia Trópico Húmedo, 26, 1-17.

Zhifeng, G., \& Thomas, B. A. (1989). A review of fossil cycad megasporophylls, with new evidence of Crossozamia Pomel and its associated leaves from the lower Permian of Taiyuan, China. Review of Palaeobotany and Palynology, 60, 205-223. 


\section{ANEXO 1}

Matriz con valores de chi-cuadrada calculada (parte superior de la diagonal) y su probabilidad (parte inferior de la diagonal) en la comparación de las curvas de estructura poblacional para diez poblaciones de Dioon holmgrenii

\section{APPENDIX 1}

Matrix of population structure curves for ten populations of Dioon holmgrenii with chi-square values (above the diagonal line) and probability (below the diagonal line)

\begin{tabular}{lcccccccccc} 
& $\begin{array}{c}\text { Rio } \\
\text { Leche }\end{array}$ & $\begin{array}{c}\text { La } \\
\text { Lima }\end{array}$ & $\begin{array}{c}\text { Ocotlán } \\
\text { Río Leche }\end{array}$ & $\begin{array}{c}\text { Rancho } \\
\text { Viejo }\end{array}$ & $\begin{array}{c}\text { Rancho } \\
\text { el Limón }\end{array}$ & $\begin{array}{c}\text { Llano } \\
\text { de León }\end{array}$ & $\begin{array}{c}\text { Cerro } \\
\text { Antiguo }\end{array}$ & $\begin{array}{c}\text { Cieneguilla } \\
\text { Cerro } \\
\text { Caballo }\end{array}$ & $\begin{array}{c}\text { San } \\
\text { Bartolomé }\end{array}$ \\
La Lima & $127.53^{\dagger}$ & 35.45 & 17.16 & 97.33 & 104.96 & 24.00 & 58.61 & 20.04 & $>181.47$ \\
Ocotlán & $<0.0001$ & & 12.23 & 54.32 & 17.41 & 68.21 & 170.91 & 28.74 & 76.11 & 36.41 \\
Rancho Viejo & 0.0001 & 0.0931 & & 27.99 & 68.21 & 47.63 & 84.69 & 18.35 & 18.35 & $>181.47$ \\
Rancho el Limón & $<0.0001$ & 0.01491 & 0.0212 & $<0.0001$ & & 74.71 & 108.62 & 47.85 & 86.56 & $>181.47$ \\
Llano de León & $<0.0001$ & $<0.0001$ & $<0.0001$ & $<0.0001$ & $<0.0001$ & & 43.07 & 21.16 & 23.94 & $>181.47$ \\
Cerro Antiguo & 0.0011 & $<0.0001$ & $<0.0001$ & $<0.0001$ & $<0.0001$ & $<0.0001$ & & 65.44 & 34.38 & $>181.47$ \\
Cieneguilla & $<0.0001$ & 0.0002 & 0.0105 & 0.0026 & $<0.0001$ & 0.0035 & $<0.0001$ & & 17.50 & $>181.47$ \\
Cerro Caballo & 0.00549 & $<0.0001$ & $<0.0001$ & 0.4618 & $<0.0001$ & 0.0012 & $<0.0001$ & 0.0144 & & $>181.47$ \\
San Bartolomé & $<0.0001$ & $<0.0001$ & $<0.0001$ & $<0.0001$ & $<0.0001$ & $<0.0001$ & $<0.0001$ & $<0.0001$ & $<0.0001$ & \\
\hline
\end{tabular}

†Valor chi-cuadrada de tablas con siete (ocho menos un estadio) grados de libertad: $\alpha 0.01=18.475$ y $\alpha 0.05=14.067$.

${ }^{\dagger}$ Chi-square value of tables with seven (eight minus one life-stage) degrees of freedom: $\alpha 0.01=18.475$ and $\alpha 0.05=14.067$.

ANEXO 2

Matriz de coeficientes de semejanza de estadios en diez poblaciones de Dioon holmgrenii

APPENDIX 2

Matrix of similarity coefficients of life stages in ten populations of Dioon holmgrenii

\begin{tabular}{lcccccccccc} 
& $\begin{array}{c}\text { Río } \\
\text { Leche }\end{array}$ & $\begin{array}{c}\text { La } \\
\text { Lima }\end{array}$ & Ocotlán & $\begin{array}{c}\text { Rancho } \\
\text { Viejo }\end{array}$ & $\begin{array}{c}\text { Rancho } \\
\text { el Limón }\end{array}$ & $\begin{array}{c}\text { Llano } \\
\text { de León }\end{array}$ & $\begin{array}{c}\text { Cerro } \\
\text { Antiguo }\end{array}$ & $\begin{array}{c}\text { Cieneguilla } \\
\text { Cerro } \\
\text { Caballo }\end{array}$ & $\begin{array}{c}\text { San } \\
\text { Bartolomé }\end{array}$ \\
Río Leche & & 0.49 & 0.62 & 0.64 & 0.59 & 0.52 & 0.65 & 0.63 & 0.47 & 0.51 \\
La Lima & 22.73 & & 0.83 & 0.69 & 0.72 & 0.53 & 0.28 & 0.67 & 0.68 & 0.79 \\
Ocotlán & 7.26 & 6.37 & & 0.78 & 0.78 & 0.59 & 0.37 & 0.77 & 0.70 & 0.87 \\
Rancho Viejo & 6.76 & 13.02 & 4.84 & & 0.68 & 0.73 & 0.38 & 0.84 & 0.78 & 0.77 \\
Rancho el Limón & 11.69 & 15.03 & 7.53 & 8.59 & & 0.76 & 0.46 & 0.82 & 0.61 & 0.72 \\
Llano de León & 11.28 & 23.00 & 11.44 & 4.84 & 8.28 & & 0.39 & 0.88 & 0.72 & 0.61 \\
Cerro Antiguo & 1.44 & 29.73 & 11.43 & 11.51 & 11.67 & 12.18 & & 0.45 & 0.27 & 0.30 \\
Cieneguilla & 7.00 & 13.73 & 5.03 & 2.78 & 4.58 & 2.49 & 8.83 & & 0.70 & 0.69 \\
Cerro Caballo & 45.85 & 32.95 & 30.17 & 22.87 & 28.67 & 26.43 & 52.25 & 25.76 & & 0.74 \\
San Bartolomé & 24.29 & 11.04 & 7.57 & 16.24 & 13.13 & 25.83 & 28.92 & 15.98 & 27.48 & \\
\hline
\end{tabular}

Valores de ISct parte superior de la diagonal y distancia euclidiana al cuadrado parte inferior de la diagonal.

ISct values upper part of the diagonal and Euclidean distance squared lower part of the diagonal. 